\title{
Masked Near-Haploidy
}

National Cancer Institute

\section{Source}

National Cancer Institute. Masked Near-Haploidy. NCI Thesaurus. Code C114475.

A chromosomal abnormality in which a near-haploid cell line is masked by a co-existing hyperdiploid line. 